\title{
SEMICONVECTION AND THE RR LYRAE VARIABLES
}

\author{
A. V.SWEIGART and P. DEMARQUE \\ Yale University Observatory, U.S.A.
}

\section{Introduction}

Theoretical computations (Hoyle and Schwarzschild, 1955; Faulkner, 1966; Iben and Rood, 1970; Demarque and Mengel, 1971a, b) have identified the horizontal-branch stars in globular clusters with the evolution phase in which helium burns within a convective core and hydrogen burns in a shell outside the convective core. Most computations for such double-energy-source models have indicated that the evolution proceeds smoothly on a nuclear time scale during the horizontal-branch phase, leading to small predicted rates of change in the RR Lyrae pulsation period (Iben and Rood, 1970). Sweigart and Demarque (1972) have recently considered the effects of semiconvection on the horizontal-branch evolution of typical Population II stars and have suggested that changes in the composition distribution within the core may occur on a time scale considerably shorter than the nuclear time scale during the phase immediately preceding core-helium exhaustion. It has been found that the composition distribution generated by the growth of a semiconvective zone in the layers surrounding the convective core can become unstable when $Y_{c}$, the helium abundance within the convective core, decreases below roughly 0.12 . The changes in the internal structure caused by this instability result in relatively rapid movement of the models in the HR diagram and consequently produce large predicted rates of change in the RR Lyrae pulsation period. The possibility that RR Lyrae period changes may be associated with the behavior of the semiconvective zone has been previously suggested by Schwarzschild (1970). A similar instability may occur in the late core-hydrogen burning phase for stars around $10 M_{\odot}$. Percy (1970) has noted the coincidence of $\beta$ Cephei stars with stellar models containing semiconvective zones. It is tempting to suggest that such an instability in the semiconvective zone could also be related to the $\beta$ Cephei phenomenon.

The principal purposes of the remaining sections are as follows: (1) to discuss the horizontal-branch evolution of a $0.65 M_{\odot}$ Population II star (Section 2), (2) to describe the instability in the core-composition distribution (Section 3) and (3) to illustrate the rates of period change which this instability produces for this star (Section 4).

\section{Horizontal-Branch Evolution of a $0.65 M_{\odot}$ Star}

A $0.65 M_{\odot}$ star with the envelope composition parameters $X=0.732$ and $\mathrm{Z}=0.001$ has been evolved from the initial horizontal-branch phase to the point of helium exhaustion in the core (Sweigart and Demarque, 1972). The evolutionary track, presented in Figure 1, was computed for an initial core mass of $0.468 M_{\odot}$. 


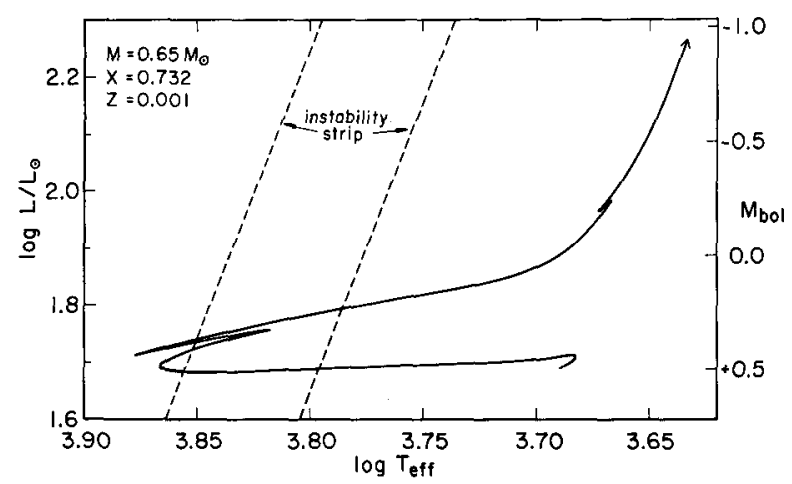

Fig. 1. The horizontal-branch track for a $0.65 M_{\odot}$ star with the envelope composition parameters $X=0.732$ and $Z=0.001$. Approximate limits for the instability strip taken from Iben and Rood (1970, Figure 7) are indicated by the dashed lines.

The model construction has been performed with generally the same numerical techniques as those adopted by Sweigart and Demarque (1972). Radiative opacities were obtained from Cox and Stewart (1970), and a mixing-length equal to 1 pressure scale height was used in treating the surface convection zone. The reaction ${ }^{12} \mathrm{C}(\alpha, \gamma){ }^{16} \mathrm{O}$ was not included in determining the helium burning rate. Lauterborn et al. (1972) have shown that the basic features of the horizontal-branch evolution are insensitive to the omission of this reaction.

The investigations of Schwarzschild and Härm (1969), Paczyński (1970), Castellani et al. (1971b) and Demarque and Mengel (1972) have demonstrated that stars with masses less than several solar masses will form a semiconvective zone in the region adjacent to the convective core during the phase of core-helium burning. The method proposed by Robertson and Faulkner (1972) has been employed to treat the occurrence of semiconvection in the present $0.65 M_{\odot}$ star. Failure to incorporate such a treatment of semiconvection can lead to a substantial underestimate of the lifetime of the horizontal-branch evolution and of the length of the horizontal-branch track in terms of $\log T_{\text {eff }}$ (Demarque and Mengel, 1972; Sweigart and Demarque, 1972).

During the initial horizontal-branch evolution of this $0.65 M_{\odot}$ star the mass content of the convective core grows as a result of convective overshooting (Castellani et al., 1971a). The reason for this growth is the necessity to fulfill the condition of convective neutrality just inside the convective-core edge (Schwarzschild, 1958). This stage of convective overshooting lasts for $17 \times 10^{6} \mathrm{yr}$ during which $Y_{\mathrm{c}}$ is reduced to a value of 0.82 and during which the convective-core mass increases from 0.105 to $0.147 M_{\odot}$. The interior composition distribution in the models of this stage therefore consists of a somewhat helium-depleted convective core which is separated by a sharp composition discontinuity from an exterior region of nearly pure helium in radiative equilibrium.

For $Y_{\mathrm{c}}<0.82$ continued expansion of the convective core is no longer capable of preserving neutrality on the inner side of the convective-core edge due to a charac- 
teristic turning up of the radiative gradient $\nabla_{\mathrm{rad}}(=\mathrm{d} \log T / \mathrm{d} \log P)$ with increasing values of $M_{\mathbf{r}}$ (Castellani et al., 1971b; Demarque and Mengel, 1972). The star resolves this dilemma by creating a semiconvective zone in the layers immediately outside the convective core. In this semiconvective zone the helium abundance $Y$ varies smoothly by the amount needed to enforce the requirement of convective neutrality.

During the evolution to the blue along the main part of the horizontal-branch track the mass in the semiconvective zone increases while the mass in the convective core remains almost constant at a value of $0.15 \mathrm{M}_{\odot}$. At the bluest point of the main horizontal-branch track $\left(\log T_{\text {eff }}=3.866, \log L / L_{\odot}=1.692\right) Y_{\mathrm{c}}$ has decreased to 0.28 . The semiconvective zone reaches its maximum extent shortly after the star begins its initial return to the red. The interior composition distribution at this stage is depicted in Figure 2. The semiconvective zone in this figure encompasses the mass between $M_{\mathrm{r}}=0.148$ and $0.256 M_{\odot}$ and thus contains about $0.11 M_{\odot}$. Following this stage the

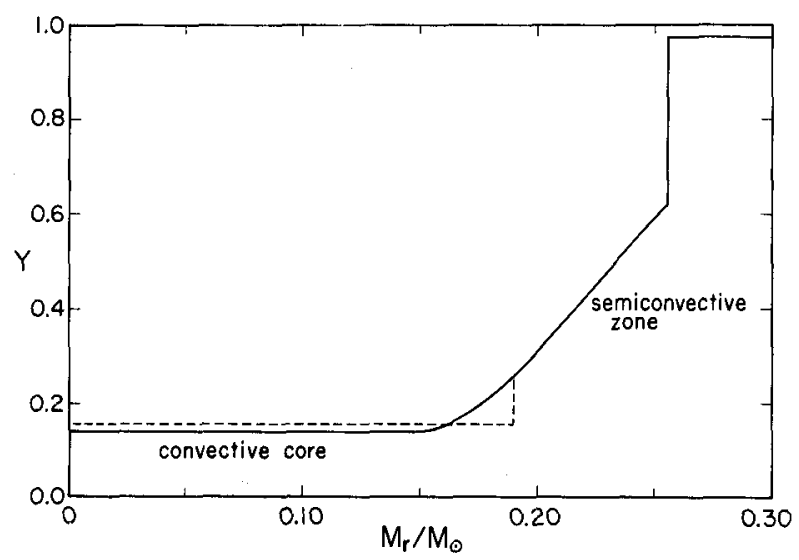

Fig. 2. The composition distribution within the core of a Population II horizontal-branch star of $0.65 M_{\odot}$ at the stage of maximum extent of the semiconvective zone. The dashed line represents qualitatively the modification in the composition profile produced by overshooting at the convective-core edge.

outer edge of the semiconvective zone recedes, leaving behind a region of varying helium abundance that is stable against convection by a small amount. When $Y_{\mathrm{c}}$ reaches a value of 0.12 , the composition distribution within the core undergoes a strong perturbation caused by the instability described in Section 3. It is this perturbation which leads to the abrupt blueward shift of the track in Figure 1 at $\log T_{\text {eff }}=$ 3.818 .

\section{Instability of the Composition Distribution Within the Core}

One might normally expect that, subsequent to the stage shown in Figure 2, there would be a gradual depletion of the remaining helium supply within the convective core. Some reasons exist, however, for suspecting that the evolution just prior to core-helium exhaustion might not proceed in such a smooth fashion (Sweigart and 
Demarque, 1972). Assume that the mass content of the convective core increases somewhat because of convective overshooting and that consequently $Y_{\mathrm{c}}$ is enriched by an amount $\delta Y$. Even a small positive perturbation $\delta Y$ will enhance the rate of energy generation from the triple- $\alpha$ process by a relatively large amount if $Y_{\mathrm{c}}$ is sufficiently small. A significant enhancement in the helium burning rate might then raise the radiative gradient $\nabla_{\mathrm{rad}}$ in the neighborhood of the convective-core edge, thereby maintaining the convective overshooting. Such a readjustment of the models would thus represent an instability in the core-composition distribution that could permit a considerable growth in the convective-core mass. The convective overshooting needed to initiate this instability is provided by the increase in the radiative opacity that occurs with the depletion of $Y_{\mathrm{c}}$ (Castellani et al., 1971a). The driving mechanism is due to the progressively greater effect which a perturbation $\delta Y$ of fixed magnitude has on the total helium-burning rate as $Y_{\mathrm{c}}$ is lowered. These general arguments have been confirmed by a stability analysis and by model computations, both of which we now discuss.

To perform a stability analysis of the core-composition distribution requires a knowledge of the way a model readjusts in response to a perturbation $\delta Y\left(M_{\mathrm{r}}\right)$ in the helium abundance. An expansion of the convective core due to overshooting will modify the composition distribution in the manner indicated qualitatively by the dashed curve in Figure 2. At each point we define $\delta Y\left(M_{\mathrm{r}}\right)$ to be the difference between the dashed and solid curves of Figure 2. Outside the point of maximum overshooting $\delta Y\left(M_{r}\right)$ is set equal to 0 .

The set of equations governing the readjustment is obtained by expanding the four stellar structure equations to first order in terms of the perturbations $\delta r, \delta L_{r}, \delta P, \delta T$ and $\delta Y$. The basic procedure is to impose a prescribed perturbation $\delta Y\left(M_{r}\right)$ on a model and to determine from these linearized equations the resulting perturbations in the remaining physical variables. This solution immediately yields the perturbation in the ratio of the radiative to adiabatic gradients, i.e. $\delta\left(\nabla_{\mathrm{rad}} / \nabla_{\mathrm{ad}}\right)$.

An essential ingredient for choosing the proper solution is an estimate for the time scale during which the overshooting used in specifying $\delta Y\left(M_{r}\right)$ takes place, since the solution depends directly on the assumed time scale. Castellani et al. (1971a) have investigated the efficiency of overshooting on the basis of the mixing-length theory. They provide a convenient relationship for the amount of overshooting which involves the degree of superadiabaticity and the change in composition at a convective boundary.

Stability of the core-composition distribution is defined by the requirement that $\delta\left(\nabla_{\mathrm{rad}} / \nabla_{\mathrm{ad}}\right)$ be negative in the region surrounding the convective-core edge. Such a readjustment would automatically prohibit an abrupt growth of the convective core by suppressing the overshooting. The condition for instability is consequently that $\delta\left(\nabla_{\mathrm{rad}} / \nabla_{\mathrm{ad}}\right)$ be positive in this region. Employing this criterion, we find that the models are stable roughly for $Y_{\mathrm{c}} \geq 0.12$ and unstable for $Y_{\mathrm{c}} \lesssim 0.12$.

The behavior predicted by the above stability test is displayed by the models for the $0.65 M_{\odot}$ star. In computing these models the amount of overshooting which oc- 
curs at the convective-core edge over a time step has been deternined from the relationship given by Castellani et al. (1971a, Equation $\left(5^{\prime}\right)$ ) and is thus consistent with physical conditions prevailing at the convective-core edge.

Figure 3 gives the time dependence of $Y_{\mathrm{c}}$ from the $0.65 M_{\odot}$ sequence. The value of $Y_{\mathrm{c}}$ increases from 0.12 to 0.20 over an interval of approximately $10^{6} \mathrm{yr}$ due to the movement of the convective-core edge out to the mass point $M_{r}=0.256 M_{\odot}$. The radiative gradient in the expanded convective core exhibits the characteristic mini-

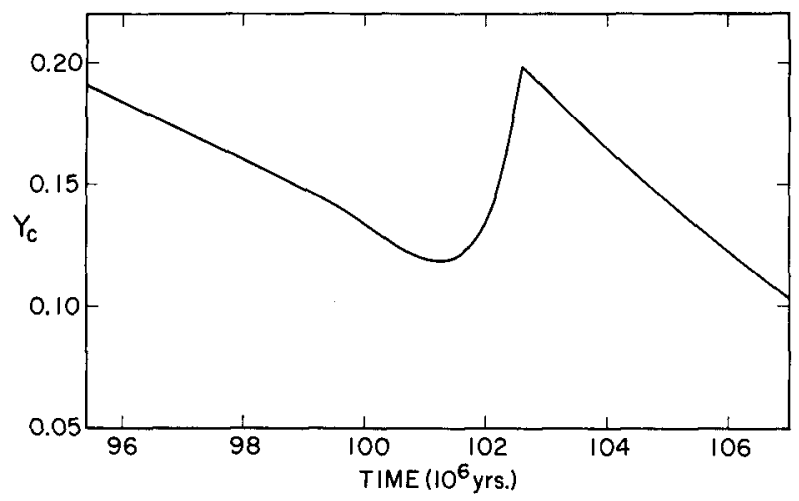

Fig. 3. Time dependence of $Y_{e}$, the helium abundance within the convective core, prior to corehelium exhaustion in a Population II horizontal-branch star of $0.65 M_{\odot}$. The time scale gives the time elapsed since the initial horizontal-branch phase.

mum referred to in Section 2 (Demarque and Mengel, 1972). The increase in $Y_{\mathrm{c}}$ is terminated when this minimum falls below the adiabatic gradient. The basic features of this behavior resemble those of the 'core breathing' phenomenon discussed by Castellani et al. (1971b) and Demarque and Mengel (1972) except that in the present case the nuclear time scale exceeds the overshooting time scale. During the $Y_{c}$ increase the overshooting time scale governs the rate of $Y_{c}$ enrichment.

\section{Predicted Rates of Change in the RR Lyrae Pulsation Period}

As demonstrated in Section 3, $Y_{c}$ does not decrease monotonically near the end of the horizontal-branch phase but rather undergoes at least one period of relatively rapid enrichment. Such a variation in $Y_{c}$ represents a significant change in the internal structure and thus strongly influences the shape of the theoretical track in the HR diagram. The increase in $Y_{c}$ around the time of $102 \times 10^{6} \mathrm{yr}$ in Figure 3 forces the $0.65 M_{\odot}$ models to reverse their redward movement in Figure 1 at $\log T_{\text {eff }}=3.818$ and $\log L / L_{\odot}=1.758$. The subsequent movement to the blue continues for $1.5 \times 10^{6} \mathrm{yr}$ until the $\log T_{\text {eff }}$ value reaches a maximum of 3.878. This maximum occurs once $Y_{c}$ stops increasing and is followed by yet another abrupt change in the direction of the track. The models then evolve redward through the instability strip on a nuclear time scale and approach the asymptotic branch. By the end of the track shown in Figure 1, 
$Y_{c}$ is practically exhausted, and convection within the core has almost disappeared. The fluctuation in the track around $\log T_{\text {eff }}=3.67$ is associated with a second but minor increase in $Y_{c}$ occurring at $Y_{c} \approx 0.02$. The bluest portion of the track in Figure 1 could be shifted entirely into the instability strip by repeating the present calculations for a slightly more massive star or for a slightly smaller initial core mass.

The rates of period change generated by this movement in the HR diagram are displayed in Figure 4. The formula given by Iben and Huchra (1971) for the fundamental pulsation period $P$ has been employed in deriving these rates. Essentially the same results are obtained if the quantity $1.5 \mathrm{~d} \ln R / \mathrm{d} t$, where $R$ is the radius, is instead used as an estimate for $\mathrm{d} \ln P / \mathrm{d} t$ (Iben and Rood, 1970). Figure 4 covers the time span from shortly before the star first enters the instability strip to shortly after the star leaves during its final evolution back to the red-giant branch. The time $t=86 \times 10^{6} \mathrm{yr}$, when $\mathrm{d} \ln P / \mathrm{d} t$ changes $\operatorname{sign}$, corresponds to the point of maximum effective temperature $\left(\log T_{\text {eff }}=3.866\right)$ along the main part of the horizontal-branch track. During the initial

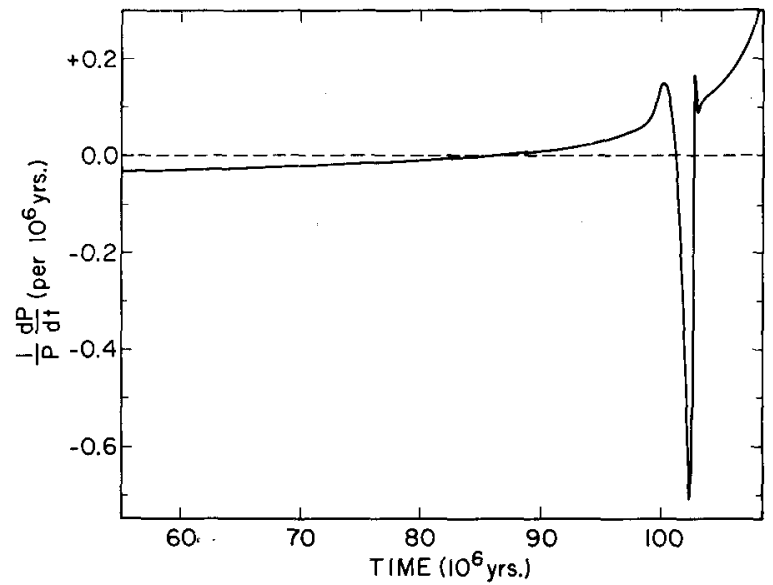

Fig. 4. The rate of change of the fundamental RR Lyrae pulsation period as a function of time for a Population II horizontal-branch star of $0.65 \mathrm{M}_{\odot}$. The time scale gives the time elapsed since the initial horizontal-branch phase.

blueward evolution $\left(t<86 \times 10^{6} \mathrm{yr}\right)|\mathrm{d} \ln P / \mathrm{d} t|$ remains less than 0.03 per $10^{6} \mathrm{yr}$ in agreement with the findings of Iben and Rood (1970). For $t>86 \times 10^{6} \mathrm{yr}$ the evolution proceeds to the red at a progressively greater rate leading to increasing values of $\mathrm{d} \ln P / \mathrm{d} t$. This is interrupted, however, for $101 \times 10^{6} \mathrm{yr} \leqslant t \leqslant 103 \times 10^{6}$ yr by a large negative fluctuation in $\mathrm{d} \ln P / \mathrm{d} t$ associated with the increase in $Y_{c}$ described in Section 3. Such $Y_{c}$ increases can thus produce values of $|\mathrm{d} \ln P / \mathrm{d} t|$ which exceed by a large amount the values which would otherwise be expected.

We will not make here a detailed comparison between the rates of period change predicted by the above computations and the observational evidence for RR Lyrae period changes due to the number of uncertainties involved. The present theoretical 
results should be regarded as somewhat preliminary partly because of the omission of the ${ }^{12} \mathrm{C}(\alpha, \gamma){ }^{16} \mathrm{O}$ reaction. In addition these results depend significantly on the accuracy of the relationship governing the efficiency of overshooting at the convectivecore edge (Castellani et al., 1971a). Consequently we emphasize the qualitative nature of both the amplitude of the $Y_{c}$ increase in Figure 3 and the precise value of $Y_{c}$ at which it begins. The existence of the $Y_{c}$ increase is, however, reasonably secure.

The observational data concerning period changes in RR Lyrae variables has been investigated by Wesselink (1973). While changes in period appear to exist for many RR Lyrae variables, Wesselink finds that the quantitative interpretation of the available data is uncertain. It is furthermore not clear whether changes in period occur in a continuous or abrupt fashion.

\section{Summary}

The results of the present study may be summarized as follows. A stability analysis shows that the composition distribution within the core will become unstable with respect to increases in the extent of the convective core near the end of the horizontalbranch phase. A growth in the convective-core mass will enrich $Y_{c}$, leading to a rapid blueward movement of the models in the HR diagram. This movement gives rise to rates of period change which are substantially greater than those caused by normal evolutionary changes on a nuclear time scale.

\section{Acknowledgements}

This research was supported in part by grant NSF GP 21345 from the National Science Foundation.

\section{References}

Castellani, V., Giannone, P., and Renzini, A.: 1971a, Astrophys. Space Sci. 10, 340.

Castellani, V., Giannone, P., and Renzini, A.: 1971b, Astrophys. Space Sci. $10,355$.

Cox, A. and Stewart, J.: 1970, Astrophys. J. Suppl. 19, 261.

Demarque, P. and Mengel, J. G.: 1971a, Astrophys. J. 164, 317.

Demarque, P. and Mengel, J. G.: 1971 b, Astrophys. J. 164, 469.

Demarque, P. and Mengel, J. G.: 1972, Astrophys. J. 171, 583.

Faulkner, J.: 1966, Astrophys. J. 144, 978.

Hoyle, F. and Schwarzschild, M.: 1955, Astrophys. J. Suppl. $2,1$.

Iben, I., Jr. and Huchra, J.: 1971, Astron. Astrophys. 14, 293.

Iben, I., Jr. and Rood, R. T.: 1970, Astrophys. J. 161, 587.

Lauterborn, D., Refsdal, S., and Stabell, R.: 1972, Astron. Astrophys. 17, 113.

Paczyński, B.: 1970, Acta Astron. 20, 195.

Percy, J. R.: 1970, Astrophys. J. 159, 177.

Robertson, J. W. and Faulkner, D. J.: 1972, Astrophys, J. 171, 309.

Schwarzschild, M.: 1958, Structure and Evolution of the Stars, Princeton University Press, Princeton.

Schwarzschild, M.: 1970, Quart. J. Roy. Astron. Soc. 11, 12.

Schwarzschild, M. and Härm, R.: 1969, Bull. Am. Astron. Soc. 1, 99.

Sweigart, A. V. and Demarque, P.: 1972, Astron. Astrophys., $20,445$.

Wesselink, A. J.: 1973, this volume, p. 104. 


\section{DISCUSSION}

Schwarzschild: The observations I think force us to look for some kind of instability or disorderliness in the horizontal branch evolution. The data on period changes seem rather convincing, I believe, in showing that superimposed on a barely detectable evolutionary period drift there exists for most RR Lyrac variables seemingly random period changes (possibly abrupt, see for example, Prager, Harvard Bulletin No. 911, 1939). Dr Sweigart has shown, I think quite persuasively, that there can exist an instability in the extent of the convective core which will seriously perturb the period. I guess that both Dr Sweigart and I regret greatly that his new instability seems to occur at most once in the instability strip, while the observations seem to require a disturbance (with more or less random signs) roughly once every 100 years.

Sweigart: Three difficulties exist in attempting to explain the random period fluctuations with a time scale of once every 100 years on the basis of the present instability of the composition distribution within the core of horizontal-branch stars. First, our $0.65 \mathfrak{M}_{\odot}$ star became unstable only once while within the instability strip. Secondly, the characteristic time scale of this instability, governed by the efficiency of overshooting at the convective-core edge, far exceeds the time scale of the period fluctuations. Thirdly, this instability only gives rise to large negative rates of period change. For these reasons Dr Schwarzschild is quite correct in emphasizing the need to search for other sources of disorderliness in horizontal-branch stars. It is not implausible that such disorderliness may be associated with the behavior of the semiconvective zone. The present instability may, however, offer a possible explanation for RR Lyrae variables which show large negative rates of period change in addition to any random period fluctuations.

Clement: Why doesn't this chemical instability repeat?

Sweigart: A second period of instability in our $0.65 \mathfrak{M}_{\odot}$ star was actually found when the helium abundance within the convective core had been reduced to 0.02 . At that time, however, this star was evolving up the asymptotic branch and therefore was no longer of interest in regard to the RR Lyrae variables. The repetition of this instability was quite mild, since the mass content of the convective core did not greatly increase. 